\title{
Herramientas y estrategias de aprendizaje en línea para la formación de posgrado en gestión de la información digital en los medios de comunicación
}

\author{
Judith Prat Sedeño \\ Archivo Municipal de Salou
}

\section{Resumen}

La tendencia respecto a la gestión de la información en cualquier empresa de medios es la de implantar sistemas que permitan no solo utilizar, compartir y desarrollar la información de esa organización, sino también incorporar como valor añadido los conocimientos de los profesionales que trabajan en ella. La finalidad última es la rentabilidad de la documentación gestionada en cuanto a adecuación, coherencia y accesibilidad. Aumentan así la calidad y la eficacia de la misma porque, en definitiva, se aporta valor a la información que se busca, organiza y transmite. Uno de los planteamientos más importantes de los licenciados que acceden a un curso de posgrado en Gestión de la información digital en los medios de comunicación debe ser el de trabajar en la integración de los contenidos de los medios informativos en las redes de comunicación de la Word Wide Web. De igual forma, el aprendizaje en línea se perfila como el más adecuado para enfrentarse a las tendencias tanto tecnológicas como sociales de personas que deben trabajar cotidianamente con las tecnologías de la información, a la vez que exige la posibilidad de interactuar con profesionales dispersos geográficamente, en una constante de comunicación sincrónica y asincrónica. Las posibilidades de la Red como un "metamedio" que permite la combinación de la escritura y la fotografía de la prensa o la publicidad, las imágenes en vídeo de la televisión y el sonido de la radio, sumadas a la concepción del receptor de la información como un ente activo en la construcción de la noticia, requieren la adaptación de los medios de comunicación tradicionales, así como profesionales formados de manera acorde. En definitiva, se pretende plantear la optimización de las herramientas de comunicación, administración, evaluación y soporte propias del e-learning en la adecuación de un diseño curricular de posgrado en Gestión de la información digital en los medios de comunicación.

Palabras clave: E-learning. Periodismo. Herramientas de comunicación. Campus virtual. Multimedia. Gestión de la información. Posgrado.

Scire. 12 : 2 (jul.-dic. 2006) 55-67. ISSN 1135-3716. 


\section{Abstract}

The tendency on the management of the information in any media company is to implant systems that allow to use, share and develop not only the information of this organization, but also to add value to the knowledge of the professionals that work in it. The last purpose is the yield of the managed documentation as regards adjustment, coherence and accessibility. Hence, its quality and efficiency increase, since value is added to the information that is searched, organized and transmited. One of the most important considerations of a graduate who enrol in a postgraduate course must be to work in the integration of the contents from the informative media to the Word Wide Web communication networks. On-line learning is outlined as one of the most suitable methods to face the new technological and social tendencies of people who must work with the information technologies every day, and in the same time it demands the possibility of interacting geographically with dispersed professionals, in a constant feature of synchronous and asynchronous communication. The possibilities of the Web as a "metamedia" that allows the combination of the writing and the photographs of press or publicity, the TV images on video and the sound of the radio, added to the conception of the receiver of the information like an active being in the construction process of the news, require the adaptation of traditional mass media. In short, the optimization of communication, administration, evaluation and support tools for e-learning in the adaptation of a postgraduate curricular design in management of the digital information in mass media is suggested.

Key words: E-learning. Journalism. Communication tools. Virtual campus. Multimedia. Information management. Postgraduate.

El ser humano está inmerso en la actualidad en el rápido y constante cambio de la tecnología y las formas de comunicación, sustentado en la compleja telaraña que es Internet; sin ella no podría entenderse el advenimiento de una terminología específica en relación con el proceso comunicativo que ha supuesto la revolución de la información en los últimos años. Así, ya en la década de los noventa, autores como Julio Cabero Almenara (1996) definían las tecnologías de información y comunicación utilizando términos ambiguos y generales, propios de la complejidad del proceso en el que estamos inmersos: inmaterialidad, interactividad, instantaneidad, innovación, elevados parámetros de calidad de imagen y sonido, digitalización, mayor influencia sobre los procesos que sobre los productos, automatización, interconexión, diversidad... Cada nueva tecnología crea un nuevo contexto social, alargando sus efectos como una sombra, a la luz de los medios. Castells (1994, p. 50), por la misma época, resultó claramente anticipador:

Estamos justo entrando en un nuevo estado en el que la cultura se remite a Cultura, habiendo sustituido la Naturaleza hasta el punto que la Naturaleza está artificialmente re-

Scire. 12 : 2 (jul.-dic. 2006) 55-67. ISSN 1135-3716. 
construida (conservada) como una forma cultural [...]. Este es el motivo por el cual la información es el ingrediente clave de nuestra organización social y por el que el fluir de imágenes entre redes constituye el hilo básico de nuestra estructura social.

¿Cómo se enfrentan al reto que comporta la gestión de la información en la era digital aquellos que facilitan y mejoran el proceso comunicativo de alcance masivo? ¿Cómo pueden los medios de comunicación mejorar y optimizar la producción, la transmisión de mensajes y la gestión de la información para perfeccionar los procesos comunicativos de los que son responsables?

La tendencia respecto a la gestión de la información en cualquier empresa de medios es la de implantar sistemas que no solamente permitan utilizar, compartir y desarrollar la información de esa organización, sino también incorporar como valor añadido los conocimientos de los profesionales que trabajan en ella. La finalidad última es la rentabilidad de la documentación gestionada en cuanto a adecuación, coherencia y accesibilidad. Aumenta así la calidad y la eficacia de la misma porque, en definitiva, se aporta valor a la información que se busca, organiza y transmite.

Siguiendo a José Luis Orihuela (2000, p. 47), el desafío profesional al que nos enfrentamos en el terreno de la comunicación no consiste simplemente en "adaptarse al cambio", ni se trata tampoco de una mera cuestión de utilización de un nuevo lenguaje. La principal exigencia no es otra que la de comprender y gestionar adecuadamente las nuevas características de los medios y de la comunicación, que adquieren el papel de protagonistas activos de la revolución tecnológica de la era digital. Desde el punto de vista formativo, esta voluntad se refleja en el contexto de formación de un Espacio Europeo de Educación Superior (EEES); sus objetivos y retos de futuro fueron expuestos oficialmente por la Declaración de Bolonia, documento suscrito en 1999 por los gobiernos de 29 países europeos y cuya culminación se espera para el año 2010.

A grandes rasgos, el EEES plantea la adquisición de competencias que permitan al individuo asumir el rol activo de evaluar la pertinencia de los conocimientos adquiridos y de los procesos seguidos. El objetivo que se persigue con ello es el de diseñar estrategias de aprendizaje optimizadas en función de los intereses individuales; la concepción del conocimiento se transforma en la de algo dinámico, lejos de la estática propia del aprendizaje tradicional, y sin duda el aprendizaje flexible y abierto que permite el e-learning, unido a las herramientas de aprendizaje colaborativo que le son propias, supone un gran avance en la consecución de los objetivos pactados desde Bolonia.

Implicaciones generales del EEES en el rol de enseñanza-aprendizaje en un posgrado de Gestión de la información digital en los medios de comunicación social:

Scire. 12 : 2 (jul.-dic. 2006) 55-67. ISSN 1135-3716. 
1. Asignaturas. Organización y desarrollo de las asignaturas de forma coordinada para la adquisición de las competencias profesionales apropiadas para cada situación profesional, de acuerdo con el perfil de los alumnos. Adecuación de la planificación del curso a la consecución de competencias profesionales, habilidades, actitudes y conocimientos.

2. Docentes. Tutorización constante en pro del aprendizaje de conocimientos y competencias, así como orientación de la docencia hacia la práctica mediante el uso de las nuevas tecnologías.

3. Alumnado. Mayor implicación en el propio proceso de aprendizaje a través del fomento de la independencia de acción frente a la autonomía. Aceptación de las competencias que se le exigirán de manera paralela a su formación específica de posgrado (mayores destrezas en el uso de idiomas, TIC, expresión escrita...).

Uno de los planteamientos más importantes de los licenciados que acceden a un curso de posgrado en Gestión de la información digital en los medios de comunicación debe ser el de trabajar en la integración de los contenidos de los medios informativos en las redes de comunicación del Word Wide Web. De igual forma, el aprendizaje en línea se perfila como el más adecuado para enfrentarse a las tendencias tanto tecnológicas como sociales de personas que deben trabajar cotidianamente con las tecnologías de la información, a la vez que exige la posibilidad de interactuar con profesionales dispersos geográficamente, en una constante de comunicación sincrónica y asincrónica. El papel que representa aquí Internet, la red de redes, es el de una vía de comunicación que lleva a los usuarios del campus virtual a los bancos y datos abiertos que se manejan en un curso de Gestión de la información digital en los medios de comunicación.

Las posibilidades de la Red como un "metamedio" que permite la combinación de la escritura y la fotografía de la prensa o la publicidad, las imágenes en vídeo de la televisión y el sonido de la radio, sumadas a la concepción del receptor de la información como un ente activo en la construcción de la noticia, requieren la adaptación de los medios de comunicación tradicionales a las características propias de las formas y contenidos de la Web, así como profesionales formados de manera acorde.

La piedra angular de la comunicación se ha desplazado hacia la producción de contenidos, la satisfacción de los usuarios y los servicios multimedia, sustituyendo a la antigua distribución parcelada de la información, característica de la era analógica. Las tecnologías digitales han conseguido procesar y emitir de forma conjunta lo fotográfico, lo cinematográfico, lo televisivo, lo escrito y lo informático, con un refuerzo mutuo entre medios y desde bases de datos multimedia que permite el servicio simultáneo a diferentes tipos de usuario, más allá de un espacio y 
un tiempo concretos. El papel que representa Internet en este entramado no es propiamente el de un medio de comunicación social, sino el de un canal de comunicación que permite un alcance masivo y personalizado, en el que cada receptor tiene a su disposición un gran volumen de información que selecciona y sobre la cual, en muchos casos, interactúa.

José Manuel de Pablos (2001, p. 258) afirma:

La red de redes no es un medio de información en el sentido de mass media sino un medio de comunicación, en el concepto de vía de comunicación, una red, valga la metáfora, pero solo como metáfora, de carreteras, calles y autopistas digitales que llevan a los usuarios desde sus monitores de trabajo hasta los bancos de datos abiertos y a la espera de la visita de los cibernautas.

Postura a la que podríamos engarzar la de Sandoval (2000), que añade:

para escribir sobre un tema no hace falta ser periodista pero, en cambio, para escribir en un medio de comunicación, sí; entonces, si consideramos a la red de redes como un entorno de comunicación, ¿por qué no están los periodistas redactando o incluso diseñando las páginas de las administraciones públicas, entidades, universidades, grandes empresas, etcétera, que circulan por la Red?

Estas últimas palabras han sido tildadas de reduccionistas por Elena Real Rodríguez (2005, p. 147) al no asumir todo contenido informativo como el único y propio fruto del ejercicio periodístico pues, según ella, no es periodista todo aquel que escribe en un medio de comunicación, ni todo contenido informativo es propiamente periodístico. Sin embargo, también sería un tanto peligroso afirmar, como esta última autora, que la función del periodista es ofrecer la información "previamente seleccionada, ordenada, interpretada". Al fin y al cabo, ¿a quién debe corresponder la labor de "interpretar", al emisor o al receptor del mensaje informativo? ¿La interpretación no incidiría en una cuestión política, lejos de la pretendida objetividad que se persigue en el quehacer periodístico? Estas son algunas cuestiones más para el debate.

Resulta particularmente interesante la mención que hace Meso Ayerdi (2002) de la formación a distancia de los periodistas: en uno de sus artículos recoge algunos datos procedentes del Primer informe anual sobre desarrollo de la sociedad de la información en España, elaborado por la Fundación Retevisión y presentado durante el año 2001, según el cual existía una necesidad urgente de formación en el sector de las tecnologías de la información y comunicación, cuyo déficit de especialistas se preveía del 13\% para el siguiente año, en función de la creciente demanda.

Desde el Departamento de Biblioteconomía y Documentación de la Facultad de Ciencias de la Información de la Universidad Complutense de Madrid se ha hecho palpable la concienciación de la necesidad de implementar en dicha universidad pública un programa de formación e-learning aplicado a la formación de posgrado en la gestión de la información en los mass media. Fruto de ello, durante

Scire. 12 : 2 (jul.-dic. 2006) 55-67. ISSN 1135-3716. 
el año académico 2005-2006 se ha puesto en marcha la primera edición del curso de posgrado en línea "Gestión de la información digital en los medios de comunicación social", apoyado en el sistema comercial de aprendizaje virtual WebCT (Web Course Tools):

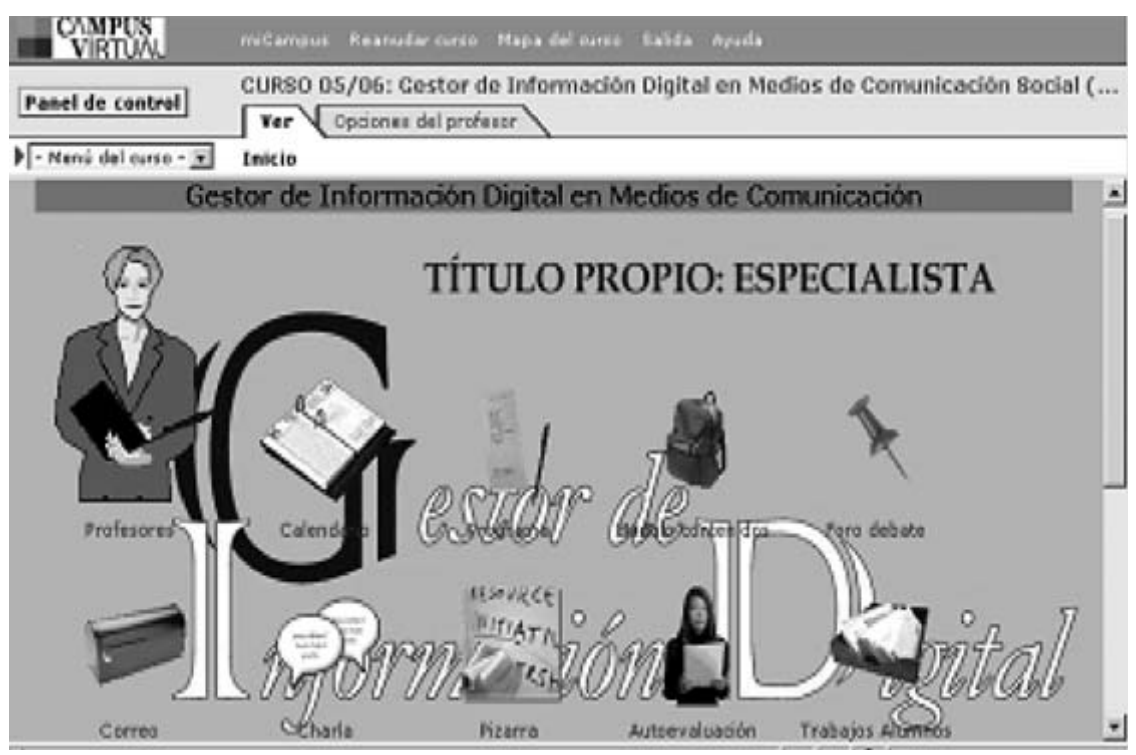

Figura 1. Menú del curso de posgrado "Gestión de la información digital en los medios de comunicación social”, primera edición, curso académico 2005-2006.

Dicho software educativo es consecuencia del trabajo del Departamento de Ciencia Informática de la University of British Columbia (Canadá). El WebCT fue presentado en el año 1997 como un producto comercial y ya ha sido utilizado por más de 2211 universidades y más de 148460 facultades. Cuenta con más de 379407 cursos y más de 10663141 estudiantes la utilizan en más de 80 países de todo el mundo. España se encuentra entre ellos, representada por las universidades de Almería, Santiago de Compostela, Barcelona o Salamanca, entre otras. Proporciona una interfaz para diseñar el aspecto del curso, herramientas educativas para facilitar el aprendizaje, la comunicación y la colaboración a nivel grupal, y un sistema de gestión administrativa del curso; además, permite la incorporación de todo tipo de materiales multimedia a través de la carga de páginas HTML. Precisamente como consecuencia de la voluntad de diseñar plataformas accesibles para docentes con poco conocimiento de HTML, la plataforma presenta algunas limitaciones y problemas de usabilidad, que se traducen en que el WebCT obliga a los profesores y administradores de los cursos a usar modelos de navegación y formatos 
de cursos predeterminados, lo que resta flexibilidad al sistema (Martín, Hassan y Martín, 2003).

Referirnos a las capacidades técnicas del software educativo con el que trabajemos es referencia obligada; sin embargo, en el diseño de un curso de posgrado lo fundamental no es la disponibilidad tecnológica, sino particularmente el modo de trabajar con la tecnología disponible al servicio del potencial usuario y los objetos de aprendizaje. De hecho, debido a la versatilidad de un posgrado virtual en Gestión de la información digital en los medios de comunicación, podemos encontrarnos con usuarios profundamente divergentes en sus motivaciones, necesidades de aprendizaje, disponibilidad de dedicación al campus o perspectivas de aplicación práctica y directa de dicho aprendizaje en sus respectivos centros de trabajo o investigación. Lo que sí que es obligado para los periodistas o futuros periodistas que trabajen en la Red es el disponer de unos conocimientos actualizados de manera constante (Salinas, 2005, p. 20).

Con esta voluntad de interacción y aprendizaje, la formación a través de Internet ofrece gran cantidad de herramientas para responder a los diferentes objetivos pedagógicos, previo conocimiento de las posibilidades de las redes en el ámbito de la enseñanza, así como de las características técnicas y pedagógicas de los servicios que nos brinda cada software educativo. Aun habiendo puesto como ejemplo de trabajo el WebCT por ser la plataforma con la que trabajamos desde la UCM, no nos vamos a centrar en ninguna en particular, sino que destacaremos los diferentes tipos de herramientas que — de forma general para cualquier plataformainteractúan en la creación, gestión y distribución de un curso on-line y cómo el posgrado en Gestión de la información digital en los medios de comunicación se sirve de ellas. Cabe distinguir cuatro grandes tipos de herramientas:

1. Herramientas de gestión y administración académica: asignación de privilegios de acceso específicos en función de la labor del individuo en el curso; administración personal de perfiles de usuario; proceso de inscripción; planes de carrera y oferta formativa.

2. Herramientas de seguimiento y evaluación: estadísticas y ficha personal por alumno; sistemas de evaluación y autoevaluación por parte de los alumnos mediante plantillas diseñadas por los docentes.

3. Herramientas de distribución de contenidos: editor de contenidos on-line; carpetas con material multimedia de docentes y alumnos; inserción de hipervínculos, imágenes y vídeos; administración de los módulos de contenidos.

4. Herramientas de comunicación y colaboración: foros de discusión por curso; sala de chat general y distribuida por módulos temáticos; formación de grupos de trabajo; comunicación con el tutor, los docentes y los compañeros; novedades y calendario del curso. 
Las dos primeras no difieren esencialmente entre un curso virtual y otro, motivo por el cual no nos detendremos en ellas. Su objetivo no es sino proporcionar los medios de gestión y administración para matricularse, consultar el expediente académico del alumno, acceder a información personal sobre este y diseñar diferentes tipologías de acceso o perfiles en función de los datos almacenados por la plataforma. Otras funciones serían las de proporcionar información al docente sobre el progreso de los alumnos en cada módulo independiente en el que esté clasificado el curso y permitir la consulta de los resultados de los tests o cuestionarios de evaluación, así como la participación de los alumnos en las herramientas de comunicación (periodicidad, tiempo de contacto...) y el seguimiento de la tutorización del proyecto de investigación, aspectos enumerados en líneas precedentes.

La tutorización del proyecto de fin de curso en el posgrado de Gestión de la información digital en los medios de comunicación social la lleva a cabo cada uno de los docentes de forma autónoma e integrada al mismo tiempo. La distribución de los contenidos — determinantes a la hora de elegir el tema de interés del alumnose basa en un primer módulo introductorio a las tecnologías de la información y la comunicación aplicadas a los mass media y otros módulos agrupados en función del medio de comunicación en el que se desarrolle la gestión digital de contenidos — prensa escrita, radio, televisión, publicidad, cine o fotografía—. Los aspectos que se tienen en cuenta en la planificación docente son los siguientes: $a$ ) ubicación del curso de posgrado de Gestión de la información digital en los medios de comunicación social en el contexto de la oferta formativa presencial y virtual de la Facultad de Ciencias de la Información de la UCM; adecuación curricular dentro de los planes de estudios; $b$ ) objetivos del curso de posgrado; $c$ ) metodología del curso; papeles que han de desempeñar el profesor y el alumno; $d$ ) contenidos del curso; utilización de material multimedia propio de las empresas periodísticas donde desarrollan sus funciones principales los docentes del curso; $e$ ) recursos externos a la plataforma virtual; bibliografía, webgrafía, bases de datos, bibliotecas virtuales, weblogs...; $f$ ) conocimientos, destrezas, actitudes y valores; $g$ ) temporización de los contenidos de acuerdo con los objetivos del curso; $h$ ) evaluación inicial sobre conocimientos previos, evaluación continua y evaluación final.

Cada uno de los módulos en los que se divide el curso reúne por término medio de tres a cuatro profesionales, los cuales ofrecen su asesoramiento individualizado a lo largo del curso en función de la elección del estudiante y de la distribución equitativa de los alumnos entre los profesores. Así, por una parte se integra el trabajo diario del alumno, que va adquiriendo conocimientos y competencias en los diferentes medios de comunicación, y el trabajo del grupo, que va desgranando cada punto y abriendo nuevos temas de discusión y recursos, ajenos a los recursos multimedia que pueda ofrecer el propio curso. Por otra parte, los docentes pueden ponerse en contacto entre sí, ya sea para la consulta de materiales o resultados en el 
aprendizaje colaborativo o para la de los materiales públicos y las herramientas de comunicación, elemento este último en el que nos vamos a centrar a continuación.

Las herramientas de comunicación son aquellas que permiten establecer una conexión tanto sincrónica como asincrónica entre los diferentes participantes del curso: docentes, alumnos o administradores. El aprendizaje virtual ofrece una flexibilidad plena a la hora llevar a cabo la comunicación; atiende a la versatilidad cada vez más necesaria en los estudios de posgrado mediante la posibilidad de establecer una comunicación tanto sincrónica como asincrónica, es decir, que emisor y receptor pueden encontrarse realizando el acto comunicativo en el mismo tiempo o en tiempos diferentes. En el posgrado que hemos emprendido desde la UCM, la totalidad de alumnos participantes trabajan en sectores vinculados a las bibliotecas, centros de documentación y/o medios de comunicación, por lo que la comunicación asincrónica se escogió como la más representativa. Otro factor que refuerza esta comunicación frente a la primera es la dificultad de sincronizar los encuentros entre profesores y alumnos cuando existe una gran diferencia horaria, como sucede en el caso de los alumnos iberoamericanos matriculados.

A continuación se enumeran las principales herramientas de comunicación que ofrece cualquier sistema de gestión de contenidos de aprendizaje basado en la Web; de todas ellas, se pueden utilizar unas u otras indistintamente, en función de los objetivos y la metodología del curso:

\section{Sincrónicas}

1. Chat. Comunicación mediante mensajes entre docentes y/o alumnos en tiempo real. Se planteará una charla general al inicio (presentación/objetivos), durante (evolución) y al final del curso (conclusiones) en una "habitación" o espacio de chateo específico para poner en contacto a los sujetos activos del curso. Además de estas charlas estipuladas, cada alumno o docente puede utilizar herramientas de comunicación asincrónicas para convocar charlas con algún interés específico, paralelo a los contenidos. De igual forma, cada intervención de los diferentes docentes que componen un módulo irá acompañada de sus correspondientes sesiones de chat al inicio y al final, con una duración estimada de una hora, de modo que los alumnos puedan realizar consultas directas al profesor y al resto de compañeros del curso. Las charlas planeadas desde la organización del curso deben estar supervisadas y dinamizadas por un moderador, que velará por un discurso ágil, ordenado y acorde con el tema principal que se exponga. De forma independiente, los tutores personales de cada alumno pueden quedar con sus dirigidos para conocer la marcha del proyecto de fin de curso. El chat adquiere una naturaleza asincrónica en el momento en que queda

Scire. 12 : 2 (jul.-dic. 2006) 55-67. ISSN 1135-3716. 
almacenado en el sistema del campus virtual y permite su recuperación y distribución para aquellos alumnos que lo soliciten.

2. Videoconferencias/audioconferencias. Emisión de vídeo y audio streaming, recurso que facilita la transmisión de clips o películas a través de la Red, regida por cada docente, sin que sea necesario descargar previamente todo el archivo. Permite la comunicación audiovisual entre docentes y estudiantes y la consulta en tiempo no real, y, precisamente por su carácter audiovisual, es especialmente recomendable preparar una videoconferencia cuando se pretende una primera toma de contacto entre el tutor o docente y el alumno. Este elemento facilita enormemente el crear un vínculo de mayor proximidad entre los sujetos activos del curso.

\section{Asincrónicas}

a) Bidireccionales

1. Foros de discusión. Constituyen la herramienta de comunicación que ha demostrado mayor dinamismo en volumen de información y frecuencia de uso en el curso de posgrado de la UCM que nos ocupa. En ellos los alumnos suministran recursos anexos, plantean sugerencias o exponen sus dudas, que son respondidas por otros alumnos o por el equipo docente; cada uno aporta algo y esta rueda colaborativa se retroalimenta diariamente hasta llegar a resultados insospechados. Esta herramienta permite el intercambio de mensajes en un plazo previamente estipulado que puede variar de días a meses. Los foros de discusión se organizan en módulos, correspondientes a cada uno de los medios de comunicación desde los cuales se plantea la gestión de la información digital, para que el intercambio de mensajes y respuestas sea más sencillo. Independientemente, existe una carpeta que recoge los comentarios generales que puedan surgir respecto al curso, no encuadrados en un módulo en concreto. Los mensajes se muestran en secuencia temporal, de modo que se fomenta el conocimiento del nivel progresivo que adquieren los alumnos y su motivación.

2. Herramientas de intercambio de archivos. Permiten que los alumnos carguen archivos de su ordenador personal y los compartan con los tutores, docentes o demás alumnos del curso de posgrado.

3. E-mail interno. Es un correo electrónico que puede ser leído o enviado exclusivamente dentro de la plataforma usada por el curso on-line. Es especialmente importante para intercambiar información con alumnos que sienten timidez a la hora de realizar ciertas preguntas y que sean vistas por el resto de sus compañeros o comentar circunstancias personales que pueden dificultar su marcha habitual en el curso.

Scire. 12 : 2 (jul.-dic. 2006) 55-67. ISSN 1135-3716. 


\section{b) Unidireccionales}

1. Tablón de anuncios. Se trata de una herramienta de comunicación unidireccional, dirigida por el profesor hacia los alumnos, cuya función radica en comunicar noticias o información de interés para el alumnado.

2. Calendario. Herramienta pensada para llevar a cabo la planificación general del curso: por ejemplo, señalar convocatorias de chats por parte de los docentes o entregas de pruebas de evaluación o prácticas.

3. Páginas personales. Cada usuario puede configurar una página personal que suele utilizarse como vía de presentación desde la cual compañeros y docentes pueden ver la fotografía del alumno, conocer su lugar de residencia, sus ocupaciones o sus intereses diversos.

Fruto de la intersección de las herramientas de comunicación sincrónicas y asincrónicas, la clase se puede organizar en grupos de trabajo que pongan en marcha actividades y debates en un espacio autónomo, mediante la creación de foros de discusión y sesiones de chat establecidos para el trabajo exclusivo de cada grupo. Los factores que configuren un grupo pueden ser diversos y estarán condicionados al acuerdo entre gestores del curso y alumnos: puede trabajarse con base en criterios temáticos, geográficos, laborales, etcétera.

Flores y Miguel (2004, p. 174) expresan contundente y sucintamente las bondades de trabajar colaborativamente en connivencia con las tecnologías de la información y la comunicación:

La nueva cultura que se está generando por el contacto con los medios digitales conducirá hacia formas originales y distintas de organizar la vida, el conocimiento y la formación. La interconexión entre ordenadores brinda la posibilidad de redefinir el concepto de inteligencia colectiva, basado en la participación en proyectos autodirigidos, fundamentados en una idea, propósito o afición común, que a la vez implica una reconceptualización de la idea de aprendizaje: se trata de pasar de un modelo de pensamiento cartesiano, basado en la idea de "pienso", a la colectiva de "pensamos".

En definitiva, con el presente trabajo se ha pretendido plantear la optimización de las herramientas de comunicación, administración, evaluación y soporte propias del e-learning para la adecuación de un diseño curricular de posgrado en Gestión de la información digital en los medios de comunicación. Se apuesta por la concepción del profesional de la información como un elemento estratégico clave en la gestión de las organizaciones y por la formación en línea como la manera más eficiente de optimizar los métodos de aprendizaje.

\section{Referencias}

Area Moreira, Manuel (1995). Medios de comunicación y escuela: la política del avestruz. // Sancho, J. M.; Millán, L. M. (eds.). Hoy ya es mañana. Tecnología y educación: un diálogo necesario. Morón (Sevilla): Publicaciones del MCEP, 1995. 95-112.

Scire. $12: 2$ (jul.-dic. 2006) 55-67. ISSN 1135-3716. 
Benito Crosetti, Bárbara de (2000). Análisis comparativo de herramientas para el desarrollo de entornos de enseñanza-aprendizaje en la WWW. Mallorca: Universitat de les Illes Balears, 2000.

Cabero Almenara, Julio (1996). Nuevas tecnologías, comunicación y educación. // Edutec: Revista Electrónica de Tecnología Educativa. 1 (feb. 1996). http://www.uib.es/depart/ dceweb/revelec1.html (2006-05-20).

Cabero Almenara, Julio (2005). Las TIC y las universidades: retos, posibilidades y preocupaciones. // Revista de la Educación Superior. 34:135 (2005) 77-100. http://www.anuies.mx/servicios/p_anuies/publicaciones/revsup/pdf/RES_135.pdf (2006-05-18).

Castells, Manuel (1994). Flujos, redes e identidades: una teoría crítica de la sociedad informacional. // Castells, M.; Flecha, R.; Freire, P.; Giroux, H.; Macedo, D.; Willis, P. Nuevas perspectivas críticas en educación. Barcelona: Paidós Ecuador, 1994. 15-50.

Centro Superior de Innovación Educativa. Aulario Virtual Universidad de Navarra. http://csie.unavarra.es/0_orok/webct/es/que_es.htm (2006-05-05).

E-learning. Grupo de Ingeniería de Organización de la UPM. http://tecnologias.gio. etsit.upm.es/elearning/ (2006-05-29).

Comezaña Portilla, Óscar; García Peñalvo, Francisco José (2005). Plataformas para la educación basada en web: herramientas, procesos de evaluación y seguridad. Salamanca: Universidad, 2005. http://tejo.usal.es/inftec/2005/DPTOIA-IT-2005-001.pdf (2006-02-03).

Flores Vivar, Jesús Miguel; Miguel Arruti, Alberto. Gestión del conocimiento en los medios de comunicación. Madrid: Fragua, 2004.

Institut Català de Tecnologia - ICT (2003). Situation and prospects for e-learning in Spain. Barcelona: ICT, 2003. http://www.teleformacion.edu/documentos/informe\%20ict.pdf (2006-03-18).

Martín Fernández, Francisco F.; Hassan Montero, Yusef; Martín Rodríguez, Óscar (2003). Limitaciones y problemas de usabilidad en plataformas de formación virtual: el caso de WebCT. // No Solo Usabilidad Magazine. 3 (jun. 2003). http://www.nosolo usabilidad.com/articulos/limitaciones_webct.htm (2006-05-09).

Martin, Gene; Middleton, Howard (eds.) (2003). Initiatives in technology education: comparative perspectives. Queensland: University of Griffith, 2003. http://teched.vt.edu/ ctte/ImagesPDFs/InitiavesInTE.pdf (2006-03-07).

Meso Ayerdi, Koldobika (2002). Un nuevo tipo de profesional llama a las puertas del periodismo: el periodista digital. // Revista Latina de Comunicación Social. 51 (jun.-sept. 2002). http://www.ull.es/publicaciones/latina/2002mesojunio5103.htm (2006-05-02).

Orihuela, José Luis (2000). Sociedad de la información y nuevos medios de comunicación pública: claves para el debate. // III Encuentro Internacional de Investigadores y Estudiosos de la Comunicación (ICOM 2000). La Habana: Universidad, 2000. 44-50. http://www.unav.es/digilab/nr/ (2006-04-26).

Pablos, José Manuel de (2001). La red es nuestra. Madrid: Paidós, 2001.

Paradela González, Luis Felipe (2001). Una metodología para la gestión del conocimiento. Tesis doctoral. Madrid: UPM, 2001.

Scire. 12 : 2 (jul.-dic. 2006) 55-67. ISSN 1135-3716. 
Platt, Richard (2006). Comunicación: de los jeroglíficos a los hipervínculos. Madrid: Edilupa, 2006.

Real Rodríguez, Elena (2005). Un intento por clarificar los actos propios del ejercicio periodístico. // Estudios sobre el Mensaje Periodístico. 11 (2005) 129-151. http://www.ucm.es/BUCM/revistas/inf/11341629/articulos/ESMP0505110129A.PDF (2006-05-18).

Salinas Ibáñez, Jesús (2000). Campus Extens. Un modelo de formación flexible en entornos virtuales. // III Congreso Internacional de Comunicación, Tecnología y Educación. Redes Multimedia y Diseños Virtuales. Oviedo. http://gte.uib.es/articulo/oviedo 001.pdf (2006-03-15).

Salinas Ibáñez, Jesús M. (coord.) (2005). Propuesta de nuevas metodologías para el desarrollo de cursos de doctorado interuniversitarios mediante el aprendizaje electrónico. Madrid: Ministerio de Educación y Ciencia, 2005. http://www.mec.es/univ/ proyectos2005/EA2005-0215.pdf (2006-06-03).

Sandoval Martín, María Teresa (2000). Los periodistas en el entorno digital: hacia el periodista multimedia. // Sala de Prensa: web para profesionales de la comunicación iberoamericanos. 22:2 (nov. 2000). http://www.saladeprensa.org/art164.htm (2006-05-30). 